Check for updates

Cite this: RSC Adv., 2019, 9, 27835

\title{
Dendrite formation in Li-metal anodes: an atomistic molecular dynamics study
}

\begin{abstract}
Luis A. Selis and Jorge M. Seminario (DD *
Lithium-metal is a desired material for anodes of Li-ion and beyond $\mathrm{Li}$-ion batteries because of its large theoretical specific capacity of $3860 \mathrm{~mA} \mathrm{~h} \mathrm{~g}^{-1}$ (the highest known so far), low density, and extremely low potential. Unfortunately, there are several problems that restrict the practical application of lithium-metal anodes, such as the formation of dendrites and reactivity with electrolytes. We present here a study of lithium dendrite formation on a Li-metal anode covered by a cracked solid electrolyte interface (SEI) of $\mathrm{LiF}$ in contact with a typical liquid electrolyte composed of $1 \mathrm{M} \mathrm{LiPF}_{6}$ salt solvated in ethylene carbonate. The study uses classical molecular dynamics on a model nanobattery. We tested three ways to charge the nanobattery: (1) constant current at a rate of one $\mathrm{Li}^{+}$per $0.4 \mathrm{ps}$, (2) pulse train $10 \mathrm{Li}^{+}$per $4 \mathrm{ps}$, and (3) constant number ions in the electrolyte: one $\mathrm{Li}^{+}$enters the electrolyte from the cathode as one $\mathrm{Li}^{+}$exits the electrolyte to the anode. We found that although the SEI does not interfere with the lithiation, the mere presence of a crack in the SEI boosts and guides dendrite formation at temperatures between 325 $\mathrm{K}$ and $410.7 \mathrm{~K}$ at any $\mathrm{C}$-rate, being more favorable at $325 \mathrm{~K}$ than at $410.7 \mathrm{~K}$. On the other hand, we find that a higher $\mathrm{C}$-rate (2.2C) favors the lithium dendrite formation compared to a lower $\mathrm{C}$-rate (1.6C). Thus the battery could store more energy in a safe way at a lower $\mathrm{C}$-rate.
\end{abstract}

Received 4th July 2019

Accepted 14th August 2019

DOI: $10.1039 /$ c9ra05067a

rsc.li/rsc-advances
Lithium dendrite growth has been extensively investigated in the last decades, ${ }^{21}$ but lithium dendrite growth is still almost inevitable during charge and discharge cycles of the battery. ${ }^{22,23}$ Lithium dendrites growth has been detected at the solid-electrolyte interphase (SEI) cracks. ${ }^{16,21}$ On the other hand, recently Zhang et al. were able to produce dendrite free electrodes using $\mathrm{FEC} / \mathrm{LiNO}_{3}$ electrolyte, ${ }^{24}$ and Mashayek et al. ${ }^{25}$ has reported Liion diffusion through the SEI of Li-metal batteries. In this work we perform MD simulations of a Li-metal anode covered by a SEI of LiF that is initially cracked, and we analyze the effects of the crack on dendrite formation at few conditions of temperature and C-rate as well as of charging protocols. We choose LiF as SEI because the large amount of theoretical and experimental studies since it is one of the main components of SEI when $\mathrm{PF}_{6}{ }^{-}$is used as counterion. ${ }^{24}$ It was also reported that the lithium ions can diffuse through $\mathrm{LiF}^{25}$ which certainly hinders to some extent the entry of lithium ions into the anode but does not completely block their entry.

\section{Methodology}

All molecular dynamics (MD) simulations are performed using the Large-Scale Atomic/Molecular Massively Parallel Simulator (LAMMPS) program developed by Plimpton et al. ${ }^{26-28}$ The initial simulation box contains a Li-metal anode covered with a SEI already cracked and an electrolyte of ethylene carbonate (EC) with a $1 \mathrm{M}$ concentration of $\mathrm{LiPF}_{6}$. We choose EC as solvent because it is the most popular solvent for current LIBs; $;^{29,30}$ one of
Department of Chemical Engineering, Department of Electrical and Computer Engineering, Department of Materials Science and Engineering, Texas A\&M University, College Station, TX 77843, USA. E-mail: seminario@tamu.edu; Tel: $+1-979-845-3301$ 
the reasons is its large dielectric constant of $90.5 .^{31,32}$ The initial simulation box size is $40.8 \AA \times 54.0 \AA \times 40.8 \AA$ and contains 640 LiF pairs, $936 \mathrm{Li}$-metal atoms, $424 \mathrm{EC}$ molecules and $28 \mathrm{LiPF}_{6}$ ion pairs. The initial geometry of the SEI is taken from an earlier work, ${ }^{33}$ which was focused in a LiSi anode. Then for this work, we develop an amorphous Li-metal anode which fits geometrically with the SEI. This yields an amorphous geometry in and around the SEI. The metal $\mathrm{Li}$ and SEI has a volume of $29587 \AA^{3}$, and the electrolyte (EC and $\mathrm{LiPF}_{6}$ ) has a volume of $51980 \AA^{3}$, corresponding to a density of $1.313 \mathrm{~g} \mathrm{~cm}^{-3}$ of the EC and $1.460 \mathrm{~g} \mathrm{~cm}^{-3}$ of the electrolyte mix. The initial atomic coordinates of the EC molecules and $\mathrm{PF}_{6}{ }^{-}$ions are obtained through ab initio optimizations of the geometry using Gaussian $09 .{ }^{34}$ These geometries are used to obtain the whole electrolyte using the Packing Optimization for Molecular Dynamics Simulations (PACKMOL). ${ }^{35}$ Snapshots of the simulation box are obtained using the Visual Molecular Dynamics software (VMD) ${ }^{36}$ and 3D visualization Open Visualization Tool (OVITO). ${ }^{37}$

The cathode in this nanobattery is simplified to a controllable source of ions composed of two layers of 400 frozen strongly bonded pseudo atoms separated by 10 A (Fig. 1a and b). Li-ions are created in the empty space between the two layers of pseudo atoms. These layers also avoid the periodicity along the electric field direction during all MD simulations (equilibrations and lithiations) which are performed under an NPT ensemble. The nearest neighbor distance between pseudo atoms is set to $2 \AA$ and with a repulsive Lennard-Jones barrier $\left(\varepsilon=1.5 \mathrm{kcal} \mathrm{mol}^{-1}, \sigma\right.$ $=2.5 \AA$ ) to avoid interactions between the top and bottom of the simulation box; these barriers prevent any atom traveling from end to end, but they are sufficiently shallow to avoid bond formation, deforming the nearby structures. The layer of pseudo atoms near the electrolyte is at a distance of $2.5 \AA$ from the nearest atoms in the electrolyte. The coordinates of Li-ions created in the $\mathrm{Li}^{+}$reservoir are randomly determined (Fig. 1b).

\section{Force fields}

The interactions between Li-metal atoms and Li-metal atoms with $\mathrm{Li}^{+}$ions are modeled by a second nearest-neighbor (2NN) embedded MEAM force field, ${ }^{38,39}$ as it was used in a previous work. ${ }^{40}$ The MEAM interaction is given by the equation:

$$
E=\sum_{i}\left[F_{i}\left(\rho_{i}\right)+\frac{1}{2} \sum_{j(\neq i)} \psi_{i j}\left(R_{i j}\right)\right]
$$

where $F_{i}\left(\rho_{i}\right)$ is the embedding function, $\rho_{i}$ is the background electron density at the site atom $i$ occupies, and $\psi_{i j}\left(R_{i j}\right)$ is the pair interaction between atoms $i$ and $j$ at a distance $R_{i j}$. The background electron density $\rho$ is composed of several partial electron density terms. Each partial electron density is a function of atomic configuration and atomic electron density. The quantities used to generate the functions $F_{i}$ using density functional theory are given in Table 1 . Since $\mathrm{Li}^{+}$can be seen as a hole $\left(\mathrm{e}^{+}\right.$charge) plus a neutral $\mathrm{Li}^{0}$ as per coulombic interactions only, $\mathrm{Li}^{+}-\mathrm{Li}$ can be partitioned into a $\mathrm{Li}-\mathrm{Li}$ interaction plus $\mathrm{a} \mathrm{e}^{+}-\mathrm{Li}^{0}$ interaction. The latter reduces to zero and the former is only useful for distances smaller than $2.5 \AA$ due to the fact that $\mathrm{Li}^{+}$is reduced at distances smaller than $2.5 \AA$ from the Li-metal atoms. Therefore, we use the same MEAM force field parameters of $\mathrm{Li}-\mathrm{Li}$ interactions for the interaction between both $\mathrm{Li}$ metal and $\mathrm{Li}^{+}$at distances smaller than $2.5 \AA$.

$E_{\mathrm{c}}$ is the energy per atom, $z$ is the number of nearest neighbors in the reference structure and $r_{\mathrm{c}}$ is the cut off distance. The equilibrium distance in a lithium diatomic molecule using a MEAM force field is $2.419 \AA{ }^{40},{ }^{40}$ which is close to the experimental distance of $2.672 \AA .^{41}$

The interactions among the SEI ( $\mathrm{LiF})$ atoms are modeled with the Born-Mayer potential, ${ }^{42}$

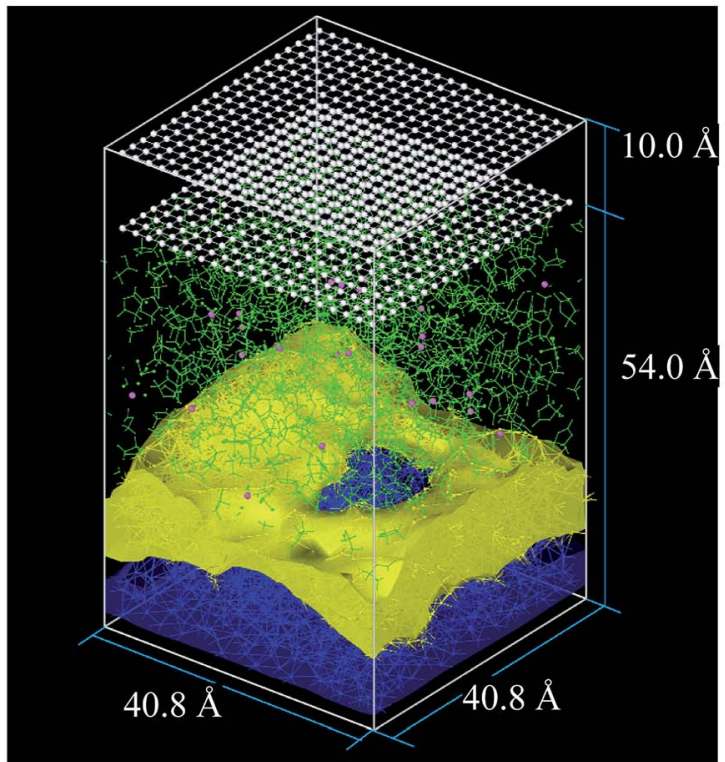

(a)

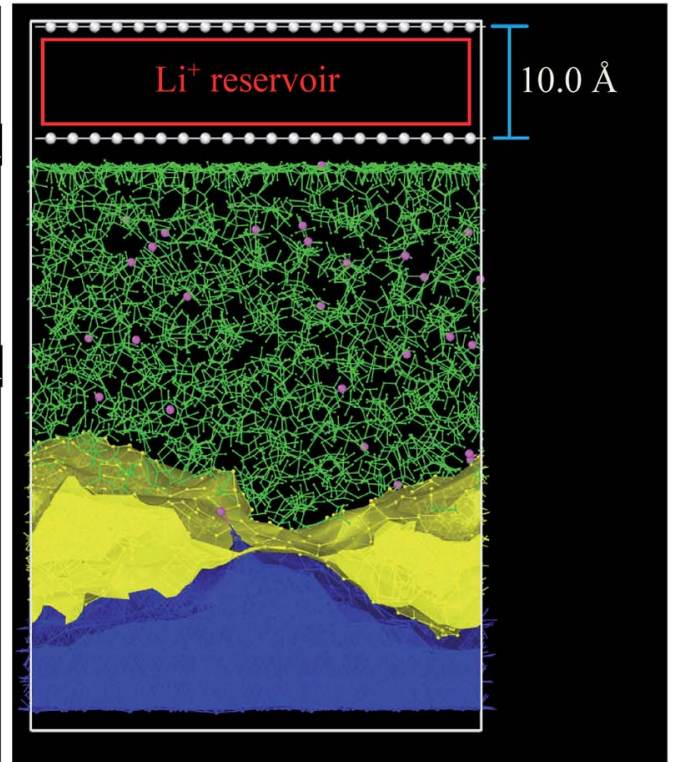

(b)

Fig. 1 (a) Initial simulation box before the equilibrium, (b) simulation box at the beginning of lithiation. Li-metal (blue), $\mathrm{Li}^{+}$(pink), EC and $\mathrm{PF}_{6}{ }^{-}$ (green), pseudo atoms (white), LiF (yellow) structure taken from an earlier work. ${ }^{33}$ 
Table 1 MEAM potential parameters for $\mathrm{Li}$ and $\mathrm{Li}^{+38}$

\begin{tabular}{ll}
\hline Parameter & Value \\
\hline Lattice type & BCC \\
$E_{\mathrm{c}}(\mathrm{eV})$ & 38.05 \\
Lattice constant $(\AA)$ & 3.509 \\
$Z$ & 8 \\
Weight $\left(\mathrm{g} \mathrm{mol}^{-1}\right)$ & 6.939 \\
$r_{\mathrm{c}}(\AA)$ & 10
\end{tabular}

$$
U\left(r_{i j}\right)=K \frac{q_{i} q_{j}}{r_{i j}}+A_{i j} \mathrm{e}^{-B_{i j} r_{i j}}-\frac{C_{i j}}{r_{i j}{ }^{6}}
$$

where $r_{i j}$ is the distance between ions $i$ and $j, q_{i}$ and $q_{j}$ are the charge of each ion, $A_{i j}, B_{i j}$ and $C_{i j}$ are parameters defined for each pair of atoms (Table 2); these parameters are taken from previous work. ${ }^{42,43}$

All the interaction between $\mathrm{Li}^{+}, \mathrm{P}, \mathrm{F}, \mathrm{C}, \mathrm{O}, \mathrm{H}$ atoms (electrolyte atoms) are modeled with an updated ReaxFF developed by Mahbubul et al. ${ }^{44}$ The ReaxFF is a bond-order based potential, including a polarizable charge calculation ${ }^{45-47}$ that allows the breaking and formation of new bonds and, in consequence, the formation or dissociation of molecules during the simulation.

Nonbonded interactions between the solvent and Li-metal, solvent and SEI, SEI and Li-metal are simulated using a Lennard-Jones (L-J) potential in conjunction with coulombic parameters (Table 3 ).

It is important to consider that the simulation times used in this work and the experimental times in commercial batteries cannot be compared directly. The simulation time and experimental times are calculated and measured in different scales; thus, we can accelerate the lithiation process and perform simulations in a reasonable computational time. We use large electric fields, which are explained in the Results section. The

Table 2 Born-Mayer potentials parameters for $\mathrm{LiF}^{42}$

\begin{tabular}{lllc}
\hline Pair & $A\left(\mathrm{~J} \mathrm{~mol}^{-1}\right)$ & $B\left(\AA^{-1}\right)$ & $C\left(\mathrm{~J}^{6} \mathrm{~mol}^{-1}\right)$ \\
\hline Li-Li & 1.03 & 1.442 & 10.0 \\
Li-F & 0.28 & 2.934 & -4.0 \\
F-F & 1.31 & 3.695 & 13.9
\end{tabular}

Table 3 Nonbonded Lennard-Jones and coulombic parameters

\begin{tabular}{lllr}
\hline Atom & $\varepsilon\left(\mathrm{kcal} \mathrm{mol}^{-1}\right)^{48,49}$ & $\sigma(\AA)^{48,49}$ & $q(\mathrm{e})^{49}$ \\
\hline $\mathrm{F}^{-}$ & 0.028 & 2.934 & -0.34 \\
$\mathrm{P}$ & 0.131 & 3.695 & 1.07 \\
$\mathrm{PF}_{6}{ }^{-}$ & & & -1.00 \\
$\mathrm{Li}^{+}$ & 0.103 & 1.442 & 1.00 \\
$\mathrm{~F}^{-}$ & 0.028 & 2.934 & -1.00 \\
$\mathrm{LiF}$ & & & 0.00 \\
$\mathrm{O}=$ & 0.210 & 2.960 & -0.65 \\
$\mathrm{O}-$ & 0.170 & 3.000 & -0.47 \\
$\mathrm{C}\left(\mathrm{sp}^{3}\right)$ & 0.105 & 3.750 & 1.10 \\
$\mathrm{C}\left(\mathrm{sp}^{2}\right)$ & 0.066 & 3.500 & 0.03 \\
$\mathrm{H}$ & 0.030 & 2.500 & 0.10 \\
Pseudo atom & 1.500 & 2.500 & 0.00
\end{tabular}

lithiation process in these simulations only takes a few ps instead of minutes or hours as it would in a real battery.

We define three protocols to charge the battery:

(1) Pulse train (PT): a pulse of $10 \mathrm{Li}^{+}$is created at random locations in the $\mathrm{Li}^{+}$reservoir every $4 \mathrm{ps}$, regardless of the number of $\mathrm{Li}^{+}$in the box or the number of $\mathrm{Li}^{+}$that are reduced at the anode. Thus, the net charge is not necessarily 0 and current is roughly constant.

(2) Constant number of ions (CI): $\mathrm{a} \mathrm{Li}^{+}$is created at random location in the $\mathrm{Li}^{+}$reservoir every moment that $\mathrm{Li}^{+}$is reduced at the anode, i.e., concerted redox reactions. Thus, the number of $\mathrm{Li}^{+}$in the box remains constant and the total charge is always 0 . Under this protocol, current increases with time because $\mathrm{Li}^{+}$ travel distance to reach the anode becomes shorter and shorter due to plating; therefore, $\mathrm{Li}^{+}$ions reach the anode and new $\mathrm{Li}^{+}$ ions are created more frequently. By comparison, other protocols keep $\mathrm{Li}^{+}$creation rate constant, and the $\mathrm{Li}^{+}$travel distance does not change the current.

(3) Constant current (CC): $\mathrm{a} \mathrm{Li}^{+}$is created at random location in the $\mathrm{Li}^{+}$reservoir every $0.4 \mathrm{ps}$. The current is closer to be constant than in the PT protocol and the net charge on the whole battery is not necessarily 0 (there must be $28 \mathrm{Li}^{+}$in the electrolyte to have 0 total charge).

In these three protocols, when a $\mathrm{Li}^{+}$reaches the anode, it transforms to a Li-metal atom. We consider $\mathrm{a} \mathrm{Li}^{+}$as having reached the anode when it is at a distance smaller than $2.5 \AA$ from any Li-metal atom.

\section{Lithiation simulations}

We consider eight cases, using several electric fields, C-rates, temperatures and protocols to determine what factors favor or restrict lithium dendrite formation. We also use different values of electric field for the $\mathrm{Li}^{+}, \mathrm{Li}, \mathrm{F}$, and the other atoms in the box. We apply strong electric fields between 1.46 and $1.5 \mathrm{~V} \mathrm{~A}^{-1}$ to $\mathrm{Li}^{+}$ to accelerate the lithiation process.

The molecular dynamics of all cases are performed under a NPT ensemble with the temperature set at $300 \mathrm{~K}$, but due to the arrival of new ions to the simulation box and the chemical reactions in the electrolyte, the temperature raises to more than $300 \mathrm{~K}$ between $325 \mathrm{~K}$ and $410 \mathrm{~K}$ in average depending of each case. These data are provided and analyzed in the result part, thus short temperature relaxation times of 30 and 50 fs (60 and 100 timesteps) are used to avoid high temperatures that could melt lithium. Also, 100 timesteps is a reasonable value for the temperature relaxation in $\mathrm{MD}$ simulations. ${ }^{50}$ For pressure relaxation at $1 \mathrm{~atm}$ we use $10 \mathrm{ps}$, which is also in the order of the reasonable value by the authors of the program and other MD specialists, ${ }^{51} 0.5$ ps (1000 timesteps). This relatively large pressure relaxation-time does not allow large changes of volume, which changes due to new few hundreds $\mathrm{Li}$ when the total sample has 7880 atoms. Therefore, volume changes are very small. The low change of volume during the whole simulation allows us to use a large pressure relaxation time of $10 \mathrm{ps}$, which is a time comparable with the times simulated under electrical field. The main parameters of the 8 cases considered are summarized in Table 4. 
Table 4 Input parameters for each case where $\mathscr{E}_{\mathrm{L}^{+}}$is the electric field for $\mathrm{Li}^{+}, \mathscr{E}_{\text {Ele }}$ is the electric field apply for $\mathrm{EC}$ and $\mathrm{PF}_{6}{ }^{-}, t_{\text {Trel }}$ is the temperature relaxation time and $Q_{E q}$ indicate whether charge equilibration is used or not

\begin{tabular}{|c|c|c|c|c|c|c|}
\hline \# & Case name & $\mathscr{E}_{\mathrm{Li}^{+}}\left(\mathrm{V} \AA^{-1}\right)$ & $\mathscr{E}_{\text {Ele }}\left(\mathrm{V} \AA^{-1}\right)$ & Protocol & $t_{\text {Trel }}(\mathrm{fs})$ & $Q_{\mathrm{Eq}}$ \\
\hline 1 & $h t_{\text {Trel }^{-}}-\mathrm{PT}$ & 1.5 & 0.5 & PT & 50 & No \\
\hline 2 & $h t_{\text {Trel }}-\mathrm{CI}$ & 1.5 & 0.5 & CI & 50 & No \\
\hline 3 & $m t_{\text {Trel }}-\mathrm{CI}$ & 1.47 & 0.5 & CI & 40 & No \\
\hline 4 & $l t_{\text {Trel }}-\mathrm{CI}$ & 1.46 & 0.5 & CI & 30 & No \\
\hline 5 & Std & 1.5 & 0.5 & $\mathrm{CC}$ & 50 & No \\
\hline 6 & $Q_{\mathrm{Eq}}-\mathrm{PT}$ & 1.5 & 0.0 & $\mathrm{PT}$ & 50 & Yes \\
\hline 7 & $Q_{\mathrm{Eq}}-\mathrm{CI}$ & 1.5 & 0.0 & $\mathrm{CI}$ & 50 & Yes \\
\hline 8 & $Q_{\mathrm{Eq}}-\mathrm{CC}$ & 1.5 & 0.0 & $\mathrm{CC}$ & 50 & Yes \\
\hline
\end{tabular}

Electric fields are applied in different ways to atoms and molecules as explained next. We do not apply electric fields to the LiF because an electric field of $1.5 \mathrm{~V} \AA^{-1}$ can break the LiF material, mainly because of the large and opposite ionic charges of \pm 1 . The coulombic attraction force and dissociation force by an electric field of $1.5 \mathrm{~V}^{-1}$ are $1.04 \mathrm{nN}$ and $2.4 \mathrm{nN}$, respectively, in a crystalline structure. The LiF SEI is already cracked with an amorphous structure and several broken bonds, and its structure dissociates easily. We apply an electric field of $0.5 \mathrm{~V}^{-1}$ to the $\mathrm{EC}$ and $\mathrm{PF}_{6}{ }^{-}$in cases 1-5, which do not have charge equilibration (no $Q_{\mathrm{Eq}}$ ) because a stronger field (e.g., of $1.5 \mathrm{~V} \AA^{-1}$ ) only increases their mobility, making the lithiation slower. Cases 6-8 have charge equilibration (using $Q_{\mathrm{Eq}}$ ) on the atoms that compose the electrolyte, thus the electrolyte atom charges can change. This characteristic helps reactions to take place and makes a more realistic simulation. A problem with performing a charge equilibration is that the computational time increases due to two reasons: the necessary additional calculations for $Q_{\mathrm{Eq}}$ to get the charge of each atom, and the charge change of a $\mathrm{Li}^{+}$from +1 to a value around +0.55 , which means the electric field applies less force to the ions. We ran the previous simulation, and based in the observed results, we estimate that would be approximately 11 times longer than cases without $Q_{\mathrm{Eq}}$; therefore, to avoid this problem in cases 6-8 (using $Q_{\mathrm{Eq}}$ ), the electric field applied to $\mathrm{EC}$ and $\mathrm{PF}_{6}{ }^{-}$is 0 . This change makes a faster lithiation possible and uses less computational resources because without electric field, the $\mathrm{EC}$ and $\mathrm{PF}_{6}{ }^{-}$vibrate less and hinder the passage of the ions less.

Case 5 is the standard or reference one due to the fact that is one of the most used methods to charge a battery. Thus, extended calculations such as the compressive stress and porosity are performed for this case.

\section{Results and Discussions}

We performed an equilibration of the box in three stages: the first stage is performed at $50 \mathrm{~K}$ for $50 \mathrm{ps}$ to eliminate any hot spots in the initial geometry. Then, in the second stage, the temperature is increased from $50 \mathrm{~K}$ to $300 \mathrm{~K}$ at a rate of $2.5 \mathrm{~K} \mathrm{ps}^{-1}$, and finally, in the third stage, the box is equilibrated at $300 \mathrm{~K}$ for $200 \mathrm{ps}$. These 3 stages are performed using the NPT ensemble with a temperature relaxation time of $50 \mathrm{fs}$ and 1 atmosphere, with a pressure relaxation time of $10 \mathrm{ps}$. Temperature noise increases as temperature increases until reaching $300 \mathrm{~K} \pm 10 \mathrm{~K}$ (3\% error margin) and we consider the sample equilibrated (Fig. 2a). The total energy per atom for the three stages of the equilibration are shown in Fig. $2 \mathrm{~b}$. The filtered average energy for all atom signals is also shown. Increasing the temperature from $50 \mathrm{~K}$ to $300 \mathrm{~K}$ minimally changes the average energy, less than $1 \%$.

The amorphous Li-metal anode stays amorphous after equilibration, but it keeps the properties of bulk Li-metal; for example, the anode has a volume of $18618 \AA^{3}$ and a density of $0.579 \mathrm{~g} \mathrm{~cm}^{-3}$, which is close to the experimental value of $0.59 \mathrm{~g} \mathrm{~cm}^{-3}{ }^{6}$ Nearest neighbor distance in the anode is $3.038 \AA$ (Fig. 3a), and the BCC structure has 8 nearest neighbors, which is the most common nearest neighbor number in this anode by the end of equilibration (Fig. 3b). To determine the number of nearest neighbors, we define the $\mathrm{Li}-\mathrm{Li}$ cutoff distance as 3.273 $\AA$, taken from the average of the experimental nearest neighbor distance in Li-metal of $3.038 \AA$ and the second-nearest neighbor distance of $3.508 \AA$.

Different protocols yield different short-circuit times. We define the short-circuit time as the time that takes to the dendrite to growth and touch the cathode as shown in Fig. 4. The charge equilibration roughly doubles the short-circuit time as it reduces the charge of any $\mathrm{Li}^{+}$, thus increasing the shortcircuit time. Therefore, we classify the 8 cases in Table 4 into two groups. Cases 1-5 do not perform charge equilibration (no $\left.Q_{\mathrm{Eq}}\right)$, and cases 6-8 do $\left(Q_{\mathrm{Eq}}\right)$. A lithium dendrite forms similarly in 7 of the 8 cases. The exception is the case of $h t_{\text {Trel }}-\mathrm{CI}$ (case 2), where the lithium dendrite grows in a very irregular pattern (Fig. 4).

Temperature and energy per atom change very differently. Temperature (Fig. 5a) is always around $300 \mathrm{~K}$ for cases $6-8$, i.e., they have similar average temperatures; therefore, these cases could dictate the protocol for better charging of the battery. For the case of the pulse train, every $4 \mathrm{ps}$, there is a peak in the temperature every $4 \mathrm{ps}$. When 10 new $\mathrm{Li}^{+}$enter to the electrolyte, they produce a strong disturbance to the box, which increases random motion and therefore, the temperature increases. Total energies per atom for each case are shown in Fig. 5b. Except for the case 2, all other seems to equilibrate to an steady state; however the strong fluctuations due to the metal-electrolyte interactions can be clearly observed. Volume variations are between $3 \%$ and $6 \%$, and variation in length of the simulation box are between $1 \%$ and $2 \%$ (Fig. $5 \mathrm{c}$ ). Thus the maximum difference of final volumes is less than $3 \%$, which means that the maximum different in final heights will be less than $1 \%$; therefore, we can assume for the sake of comparisons that all cases yield the same final height at the end of the simulations.

We compare first the cases 1-5 (no $Q_{\mathrm{Eq}}$ ) to have a better idea of how the dendrite grows in each case. We calculate the height of the highest dendrite peak corresponding to the highest $\mathrm{Li}$ atom that belongs to the dendrite (Fig. 6a). We calculate the standard deviation of heights of lithium atoms in the anode surface with respect to the anode surface (Fig. 6b) to analyze how the shape of the dendrite evolves due to the arrival of lithium atoms in each case. The dendrite height always increases (spatially), and the standard deviation of the heights of lithium atoms in the anode surface increases with time; therefore, the growth is never uniform. We calculate the 


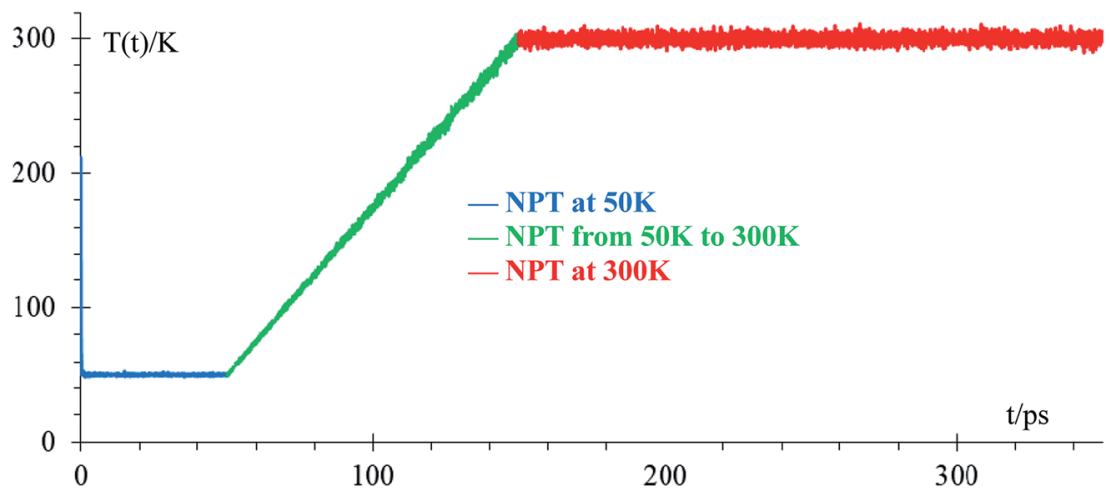

(a)

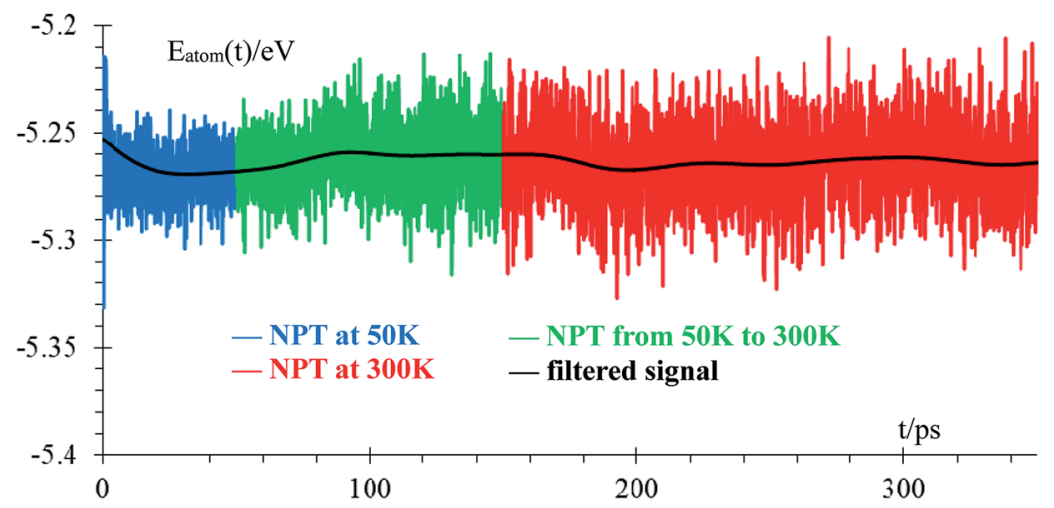

(b)

Fig. 2 Time evolution of equilibration runs: (a) temperature, (b) average energy per atom ( 7000 atoms). Filtered signal is obtained by applying a discrete time average function, $E_{n}(t)=1 / 4 E_{n-1}(t-1)+1 / 2 E_{n-1}(t)+1 / 4 E_{n+1}(t+1)$, where $E_{0}$ is the original signal and $E_{10000}$ is the filtered signal, with time intervals between samples of $50 \mathrm{fs}$ (100 steps). This averaging is used in all filtered signals, unless stated otherwise.

number of lithium atoms in the anode in each case (Fig. 6c), which is equivalent to the stored potential energy in the battery. The number of lithium atoms in the anode before the shortcircuit is different in each case; thus, each case can store a different amount of energy before the short-circuit. The standard deviation of the heights of lithium atoms in the anode surface is given by,

$$
\sigma=\sqrt{\sum_{i=1}^{n} \sum_{j=1}^{n}\left(h_{i j}-\bar{h}\right)^{2} / n^{2}}
$$

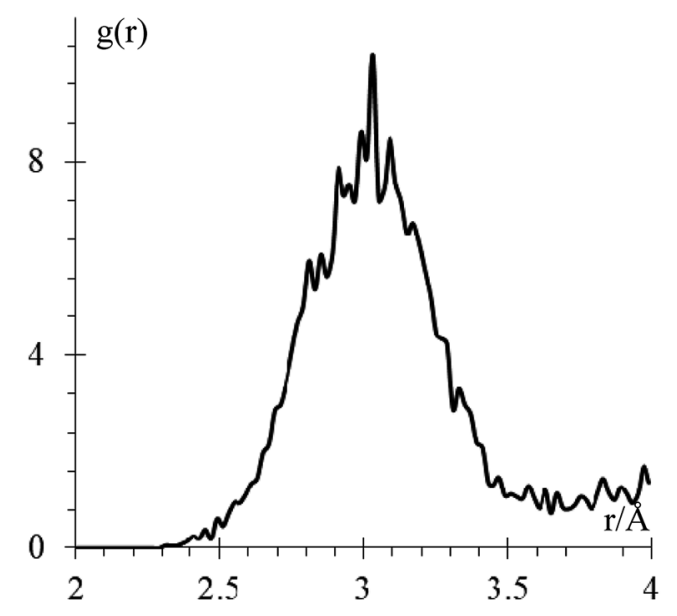

(a)

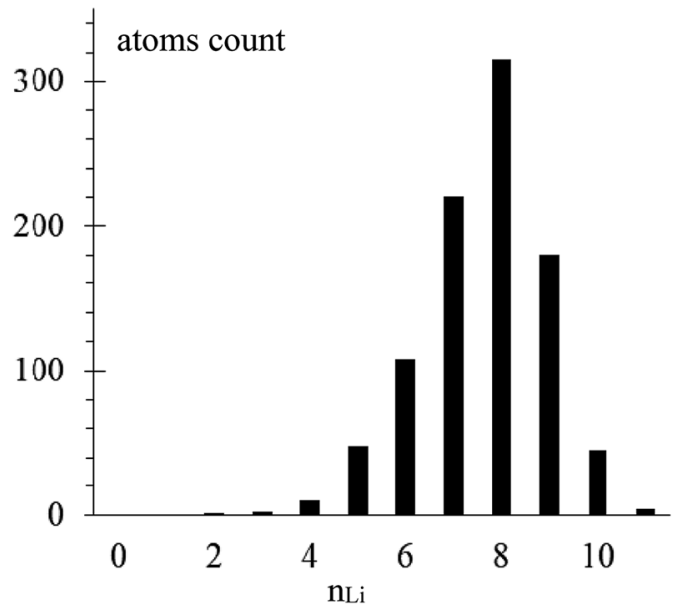

(b)

Fig. 3 (a) RDF Li-Li after equilibration (b) number of Li atoms in the anode having $n_{\mathrm{Li}}$ atom neighbors versus number $n_{\mathrm{Li}}$. There is a total of $936 \mathrm{Li}-$ atoms in the anode. 
the plane $x-y$ of the cell is divided in $n \times n(n=13)$ equal square least one Li-metal atom is on the surface of each sector. $h_{i j}$ is the sectors, thus, each square sector has a side length of $\sim 3.14 \AA$, height of the highest Li-metal in the sector $i j$, and $\bar{h}$ is the average which is larger than the Li-metal bond distance of $3.04 \AA{ }^{52}$ Thus, at height among the highest Li-metal atoms from each sector,
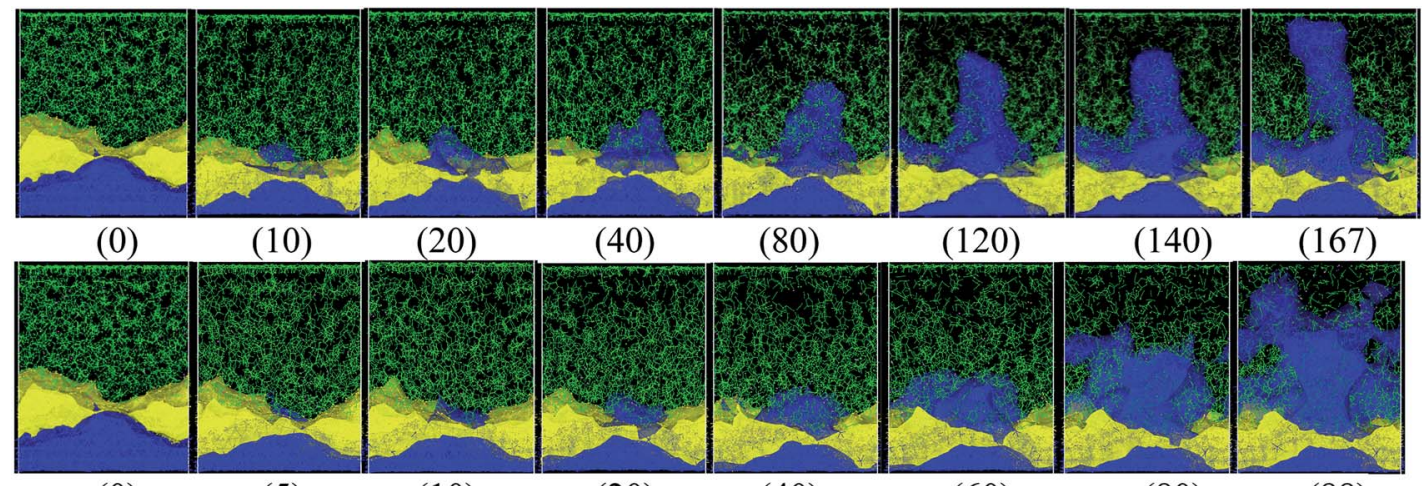

(0)

(5)

(10)

(20)

(40)

(60)

(80)

(88)
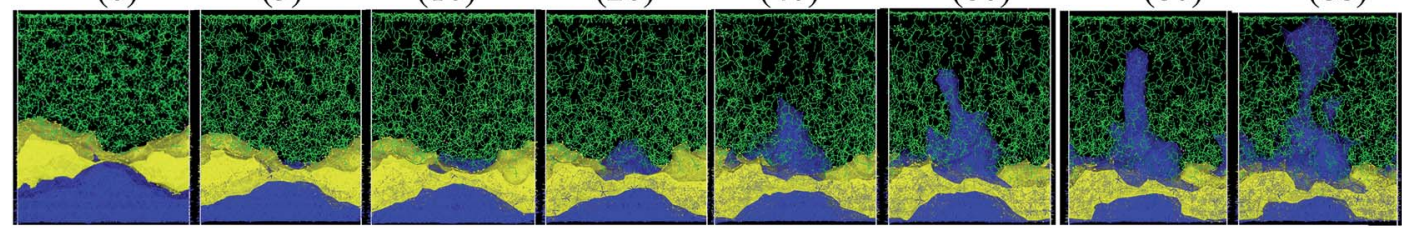

(0)

(5)

(10)

(20)

(40)

(60)

(75)
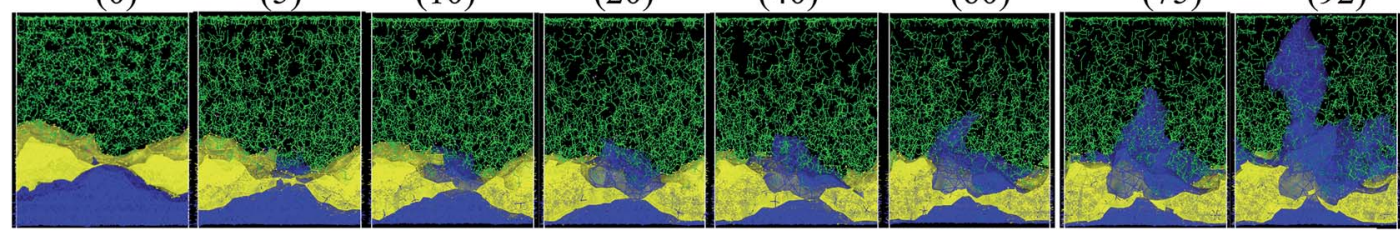

(0)

(10)

(20)

(40)

(60)

(80)

(100)

(126)
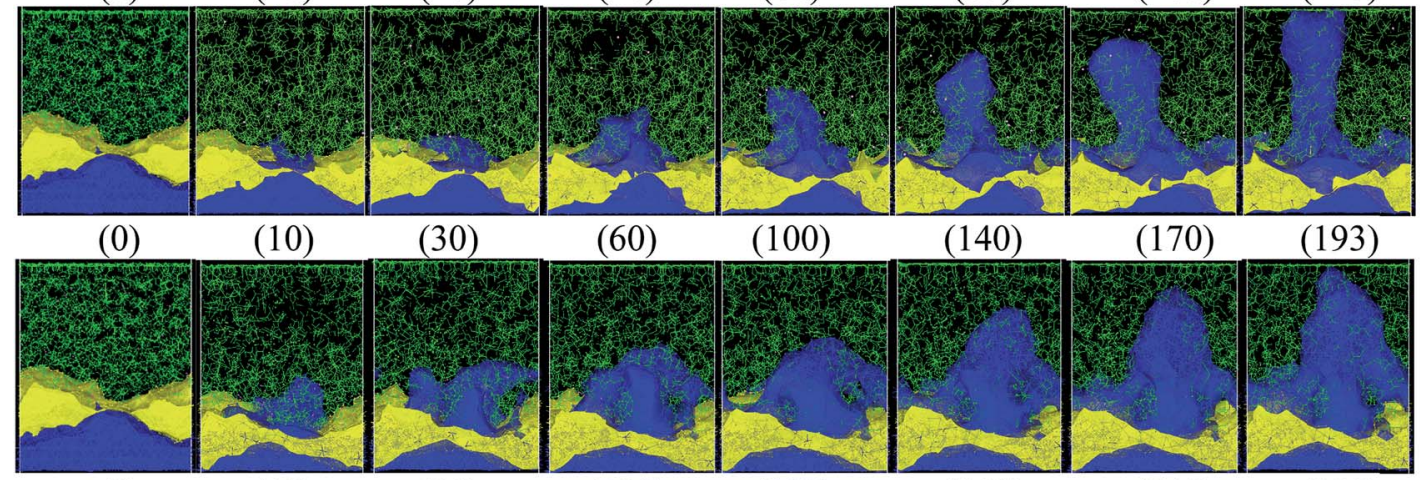

(0)

(40)

(80)

(120)
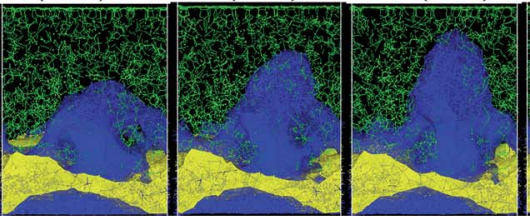

(193)
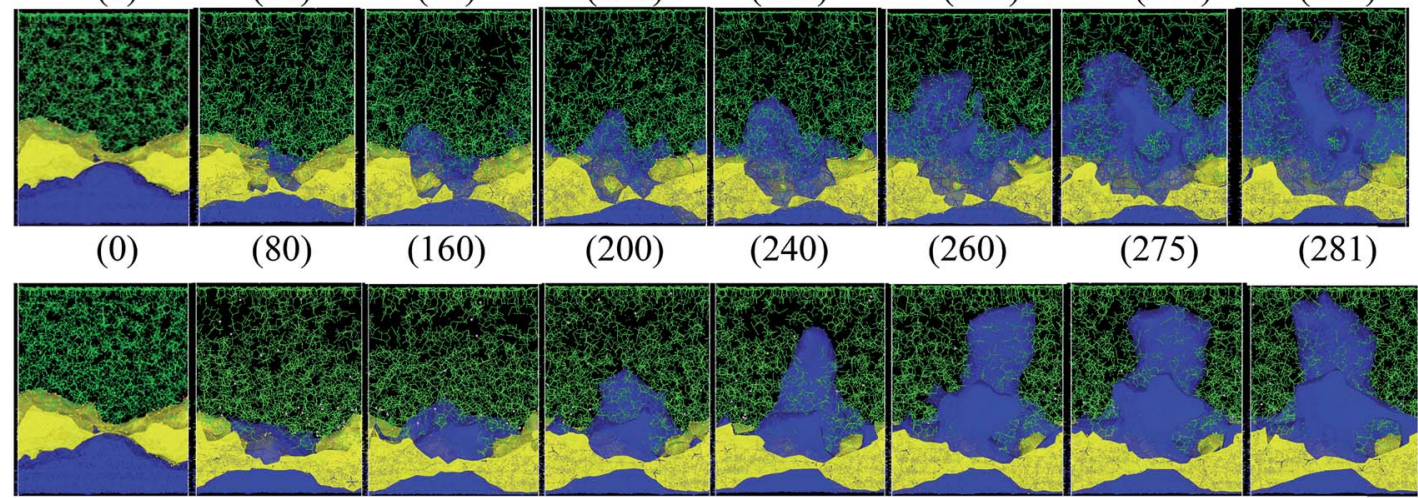

(0)

(40)

(80)

(120)
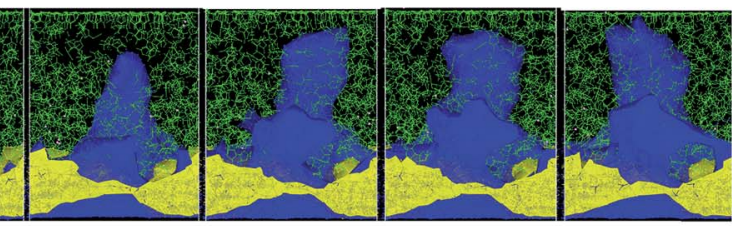

(160)

(200)

(280)

(305)

Fig. 4 Dendrite growth for cases 1-8, respectively (shown from top to bottom rows, in the same order as shown in Table 4), the label (inside parentheses) indicates the time in ps when the snapshot is taken. Color code: Li-metal (blue), Li+ (pink, very hard to see them), EC and PF 6 (green), LiF (yellow). 


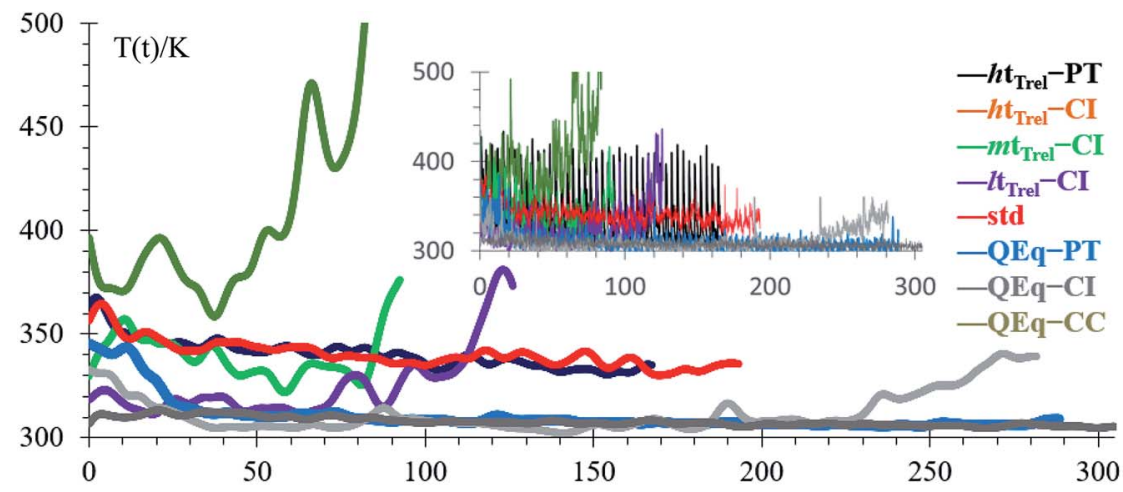

(a)

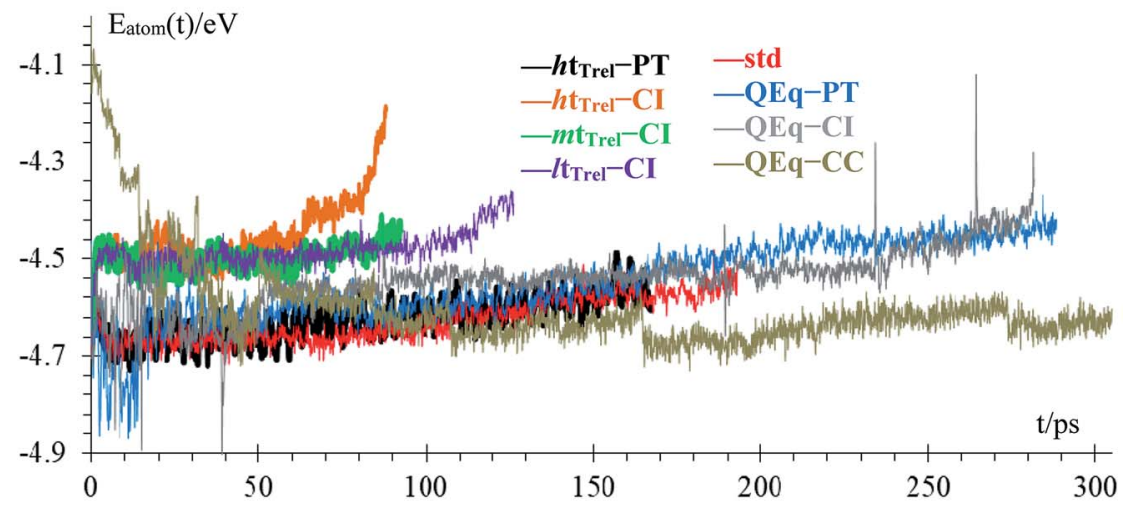

(b)

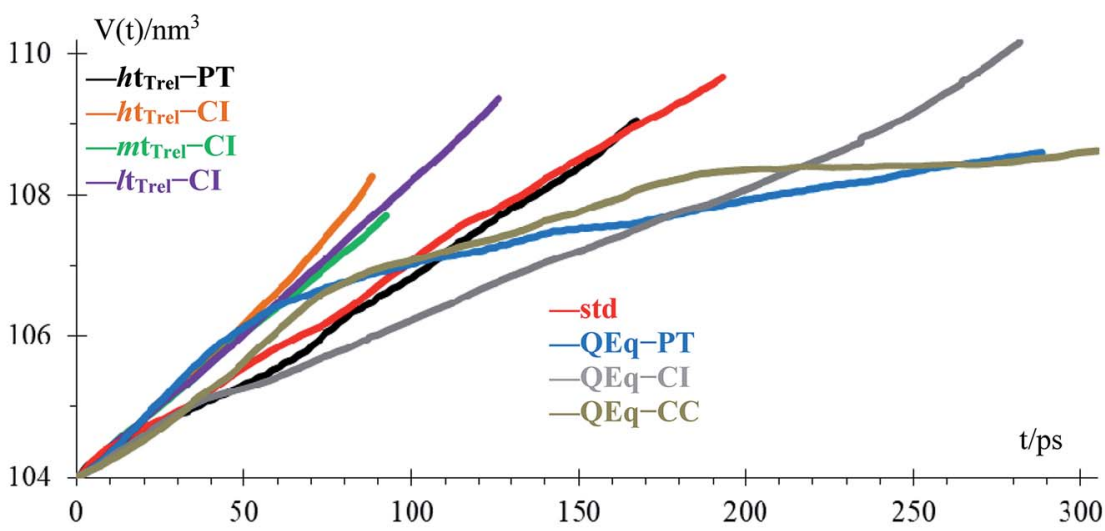

(c)

Fig. 5 (a) Low-pass filtered temperature signal; original signal in the inset, (b) energy per atom, (c) volume of the box.

$$
\bar{h}=\sum_{i=1}^{n} \sum_{j=1}^{n} h_{i j} / n^{2}
$$

Although we compare cases 1-5 (no $Q_{\mathrm{Eq}}$ ), the time comparisons are not a good way to compare them because of the different total simulation times. A better way to compare the cases 1-5 is by using the stored energy, which is equivalent to the number of lithium atoms in the anode (Fig. 7). Height of the highest dendrite peak and standard deviation of heights of lithium atoms in the anode surface tend to grow linearly respect to number of lithium atoms in the anode (stored energy) in all cases.
In cases 1-5, lithium concentrates on the LiF crack, starting the formation of a dendrite. Except for $h t_{\text {Trel }}-\mathrm{CI}$, the dendrite becomes taller and sharper; however, for $h t_{\text {Trel }}-\mathrm{CI}$, the dendrite grows very differently than in the other cases. The speed of growth of the dendrite is different for each case, determining the possible rate of charge for a safe use of the battery. We calculate the average temperature during each lithiation and the Li-metal amount before the short circuit in cases 1-5, or no $Q_{\mathrm{Eq}}$ cases (Table 5). Notice that the average temperatures in all cases is always above $325 \mathrm{~K}$, which is higher than the melting temperature of EC (310 K).

In the case of $h t_{\text {Trel }}-\mathrm{CI}$, the battery can be charged more than in the other cases before the dendrite reaches the top of the 


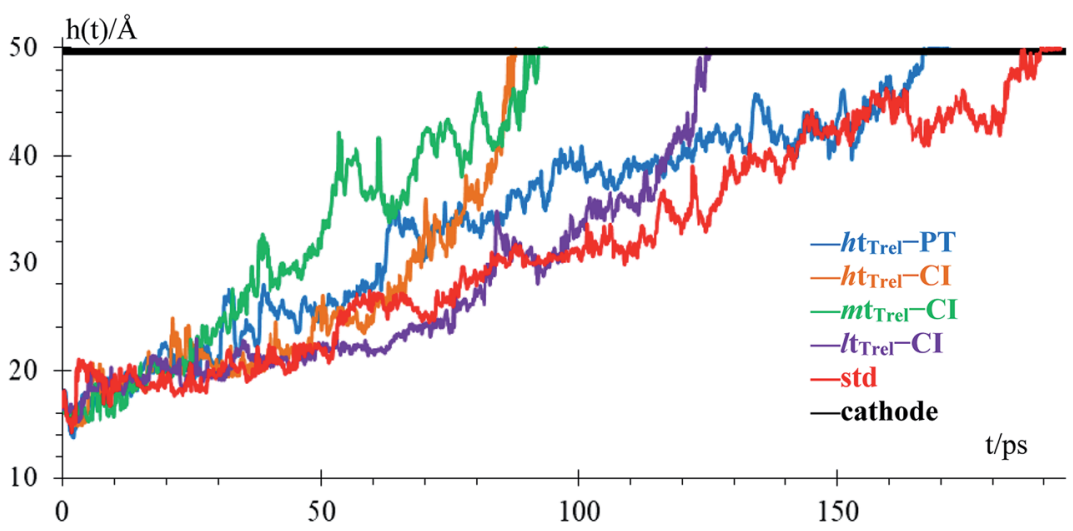

(a)

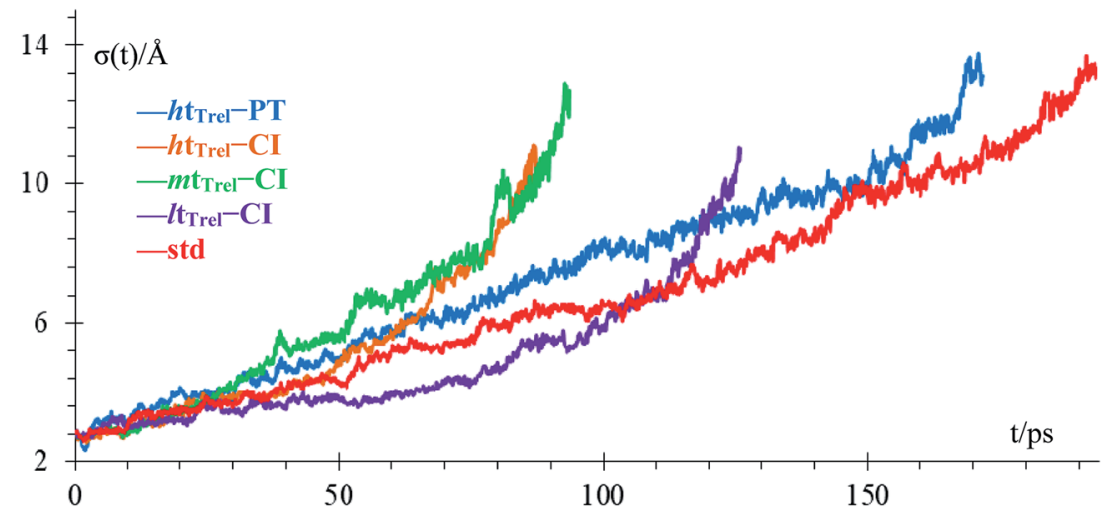

(b)

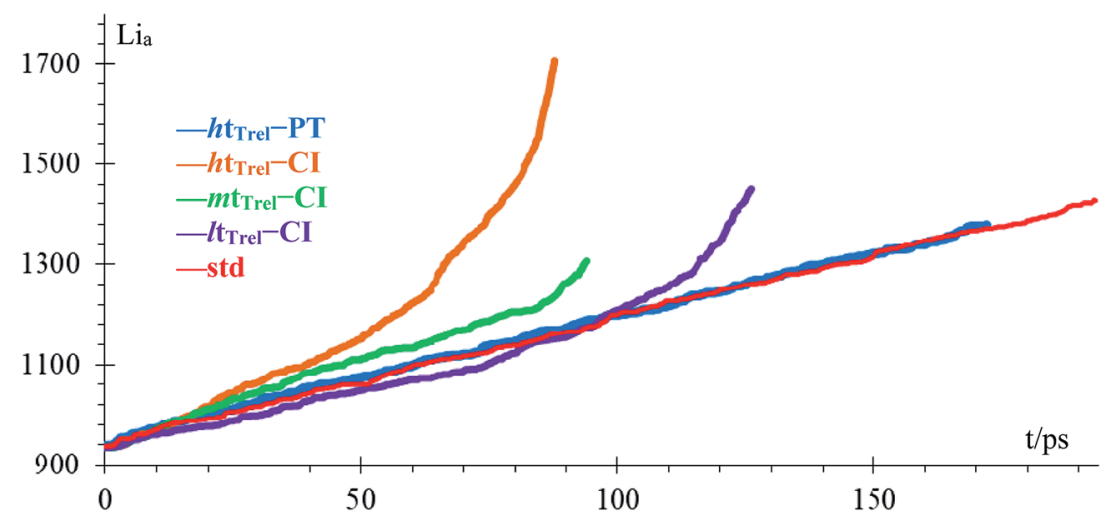

(c)

Fig. 6 Time evolution of (a) height of the highest dendrite peak, (b) standard deviation of heights of lithium atoms in the anode surface representing the dendrite formation, (c) number of $\mathrm{Li}$ atoms in the anode $\left(\mathrm{Li}_{\mathrm{a}}\right)$.

electrolyte. This is because the temperatures are higher than in any of the other cases. The velocity of all the atoms increases, and dendrite grows in all directions, making a dendrite with high volume but low height. Therefore, a high temperature could hinder dendrite formation, and the low temperature helps the lithium dendrite growth. The previous result is in accord with previous experiments ${ }^{53,54}$ and previous computational works. ${ }^{55}$

In the case of $h t_{\text {Trel }}-\mathrm{CI}, 742 \mathrm{Li}$-ions reach the anode in $88 \mathrm{ps}$ while in the case of $m t_{\text {Trel }}-\mathrm{CI}, 303 \mathrm{Li}$-ions reach the anode in 88 ps. In the case of $l t_{\text {Trel- }}-\mathrm{CI}$ in the same time period, $217 \mathrm{Li}$ atoms reach the anode. Using this data, we calculate the C-rate for cases 2-4 (Table 6). Assuming the battery has a capacity of 55 $\mathrm{A} h$, the $\mathrm{C}$-rates are scaled to a realistic value, comparing the size of the battery with a typical $55 \mathrm{~A}$ h lithium ion battery using the following relationship:

$$
\begin{aligned}
C_{\mathrm{rs}} & =C_{\mathrm{r}} \frac{L_{x}}{L_{x \mathrm{r}}} \frac{L_{y}}{L_{y \mathrm{r}}} \frac{L_{z \mathrm{r}}}{L_{z}}=C_{\mathrm{r}} \frac{141.3 \mathrm{~mm}}{40.8 \AA} \frac{137.7 \mathrm{~mm}}{40.8 \AA} \frac{64 \AA}{34 \mathrm{~mm}} \\
& =220 \times 10^{6} C_{\mathrm{r}}
\end{aligned}
$$

where $C_{\mathrm{rs}}$ is the scaled to C-rate, $C_{\mathrm{r}}$ is C-rate, $L_{x}, L_{y}, L_{z}$ are the dimensions of the nanobattery, and $L_{x \mathrm{r}}, L_{y \mathrm{r}}, L_{z \mathrm{r}}$ are the dimension of the lithium ion cell model LP 32770, yielding a scale factor of $220 \mathrm{M}$. We scale the C-rate and dendrite growth rate to 


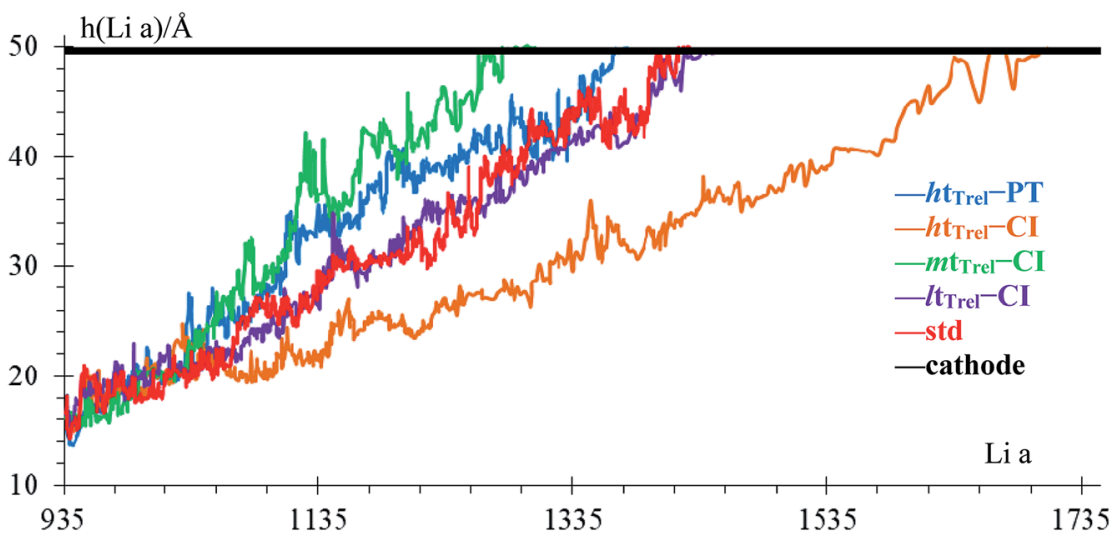

(a)

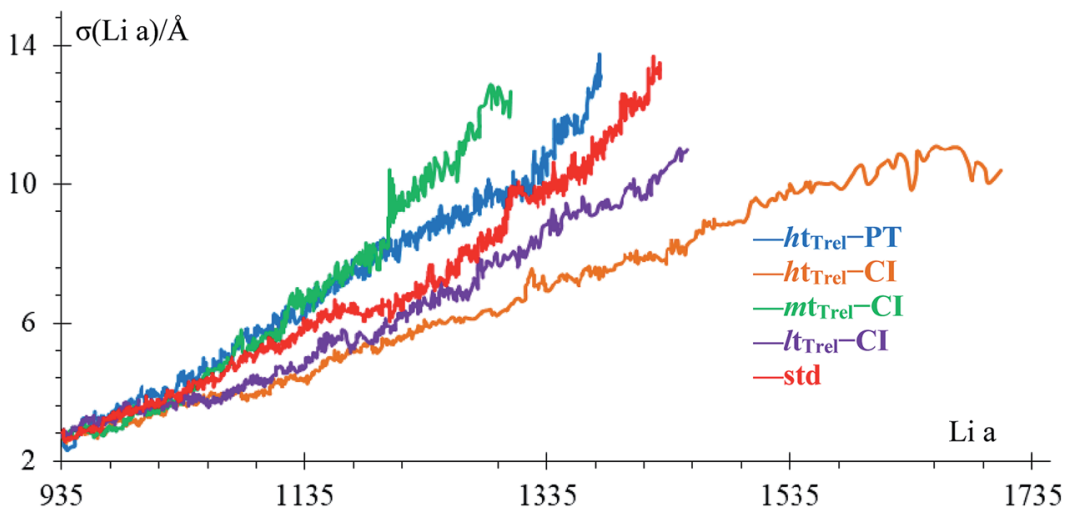

(b)

Fig. 7 (a) Height, $h$, of the highest dendrite peak vs. number of lithium atoms in the anode ( $\left(\mathrm{i}_{\mathrm{a}}\right)$, (b) standard deviation of the heights of lithium atoms in the anode surface, $\sigma$, of the dendrite formation. Standard deviation of heights of lithium atoms in the anode surface increase means that the surface of the lithium dendrite does not grow uniformly (spatially).

have a relatively good idea of how our results from nanobatteries would scale to be useful in the design of realistic size batteries. It is now a very primitive and crude scaling procedure that may eventually evolve and make our atomistic nanobattery calculations able to deal with realistic macroscopic sizes.

The case of $l t_{\text {Trel }}-\mathrm{CI}$ can be charged more than the $m t_{\text {Trel }}-\mathrm{CI}$, therefore, a high C-rate helps dendrite growth; this result is in agreement with previous experiments. ${ }^{\mathbf{5 6 , 5 7}}$ High C-rate is less important than high temperature for the grow of dendrites. Because of this, the case at $h t_{\text {Trel }}-\mathrm{CI}$ can support more lithium atoms in its anode than any other case.

Due to the fact that an electric field increases the velocity of $\mathrm{Li}^{+}$ions, lithium dendrite has a high growth rate between 17 and $40 \mathrm{~m} \mathrm{~s}^{-1}$. Therefore, to compare to experimental results, we

Table 5 Comparison of average temperatures and total lithiation times for cases 1-5 (no $Q_{\mathrm{Eq}}$ )

\begin{tabular}{lllcl}
\hline$\#$ & Case & Li count & $t_{\text {total }}(\mathrm{ps})$ & $T_{\text {ave }}(\mathrm{ps})$ \\
\hline 1 & $h t_{\text {Trel }}-\mathrm{PT}$ & 1368 & 166.8 & 340.5 \\
2 & $h t_{\text {Trel }}-\mathrm{CI}$ & 1678 & 88.4 & 410.7 \\
3 & $m t_{\text {Trel }}-\mathrm{CI}$ & 1275 & 92.5 & 339.4 \\
4 & $l t_{\text {Trel }}-\mathrm{CI}$ & 1402 & 126.2 & 325.0 \\
5 & Std & 1408 & 193.2 & 340.3
\end{tabular}

need to scale the growth rate using the same factor that we used previously to scale the C-rate (Table 7).

Scaled growth rate is in the same order of magnitude than observed in experiments. ${ }^{58}$ Actual values of the simulated scaled growth rates are a little lower than in the experiments because temperatures in our simulations are higher than $300 \mathrm{~K}$, which deter dendrite formation.

We analyze how the time evolution of the anode density changes during lithiation and whether the density in the anode core is different from that of the dendrite in the standard case. We consider a Li-metal atom to be part of the top of the anode if its height is greater than the height of highest Li-metal at time 0 . In case the Li-metal atoms are not part of the top, we consider

Table 6 Equivalent $\mathrm{C}$-rates used in cases $2-4 . n_{\mathrm{Li}}=$ number of $\mathrm{Li}$-ions reaching the anode, $I=$ total current of $\mathrm{Li}^{+}$in the battery, $t_{1}=$ time to charge $55 \mathrm{~A} \mathrm{~h}, \mathrm{C}$-rate in our battery $\left(C_{\mathrm{r}}\right)$ is $C_{\mathrm{r}}=1 \mathrm{~h} / \mathrm{t}_{1}, C_{\mathrm{rs}}=220 \times$ $10^{6} C_{r}$. C-rate analysis performed in a snapshot taken $88 \mathrm{ps}$ right after the lithiation onset

\begin{tabular}{lccclcc}
\hline Case & $n_{\mathrm{Li}}$ & $I\left(\mathrm{~A} \mathrm{~g}^{-1}\right)$ & $I(\mu \mathrm{A})$ & $t_{1}(\mathrm{~h})$ & $C_{\mathrm{r}}(\mu \mathrm{C})$ & $C_{\mathrm{rs}}(\mathrm{C})$ \\
\hline$h t_{\text {Trel }}-\mathrm{CI}$ & 742 & 13904 & 1.349 & $40,770,941$ & 0.0245 & 5.390 \\
$m t_{\text {Trel }}-\mathrm{CI}$ & 303 & 13883 & 0.551 & $99,848,511$ & 0.0100 & 2.200 \\
$l t_{\text {Trel }}-\mathrm{CI}$ & 217 & 13897 & 0.395 & $139,240,506$ & 0.0072 & 1.584
\end{tabular}


Table 7 Scaled C-rates and scaled dendrite growth rates compared with experimental results, $G_{\mathrm{r}}=\left(h_{\max }-h_{\min }\right) / t_{\text {short-circuit, }}$ where $G_{\mathrm{r}}=$ growth rate; $h_{\max }$ and $h_{\min }$ are the maximum and minimum heights (Fig. $6 a$ and $7 \mathrm{a}$ ), and $G_{\mathrm{rs}}=G_{\mathrm{r}} /\left(220 \times 10^{6}\right)$ is the estimated scaled growth rate

\begin{tabular}{|c|c|c|c|c|c|}
\hline Case & $T_{\text {ave }}(\mathrm{K})$ & $C_{\mathrm{rs}}(\mathrm{C})$ & $I(\mu \mathrm{A})$ & $G_{\mathrm{r}}\left(\mathrm{m} \mathrm{s}^{-1}\right)$ & $G_{\mathrm{rs}}\left(\mathrm{mm} \mathrm{h}^{-1}\right)$ \\
\hline$h t_{\text {Trel }^{-}}-\mathrm{PT}$ & 340.5 & 1.604 & 0.40 & 20.71 & 0.339 \\
\hline$h t_{\text {Trel }}-\mathrm{CI}$ & 410.7 & 5.390 & 1.39 & 39.28 & 0.643 \\
\hline Experiment 1 (ref. 58) & 293 & 0.481 & 1000 & & 0.92 \\
\hline Experiment 2 (ref. 58) & 293 & 0.675 & 1000 & & 1.70 \\
\hline Experiment 3 (ref. 58) & 293 & 2.000 & 1000 & & 2.18 \\
\hline
\end{tabular}

them part of the anode core regardless of their neighbors. The dendrite has very few atoms at the beginning of lithiation (10 atoms), and the graph of the density of this part has a lot of noise during the firsts ps. Density decreases with time from the beginning of the lithiation (theoretical density is $579 \mathrm{~kg} \mathrm{~m}^{-3}$ ) due to the anode becoming porous, with the most porous part being the dendrite. This porosity allows the formation of a lowdensity lithium dendrite (Fig. 8).

We analyze the compressive stress that the Li-metal anode applies to electrolyte and SEI in standard case (Fig. 9), which performs at an average temperature of $340.3 \mathrm{~K}$, and we calculate the compressive stress from

$$
\sigma_{\mathrm{N}}=\sum_{i=1}^{n} F_{z i} /\left(x_{\mathrm{size}} y_{\mathrm{size}}\right)
$$

where $n$ is the number of Li-metal atoms in the anode, $F_{z i}$ is the force applied to the lithium $i$ by all other atoms in the cell in the $z$-direction, $x_{\text {size }}$ and $y_{\text {size }}$ is the box size in the $x$ and $y$ axis respectively, and $x_{\text {size }} y_{\text {size }}$ is equivalent to the transversal area due to the dendrite growth in $z$-direction. The previous summation could be positive or negative, but we only consider the positive results (Fig. 9a) because a negative result means that the compressive stress is applied to the layer of pseudo atoms instead of the SEI.

Compressive stress could reach peaks between 1.5 GPa and 2 GPa (Fig. 9a); therefore, the SEI must support this amount of compressive stress to avoid the lithium dendrite formation in a $\mathrm{Li}^{-}$ metal anode without structural damage. We estimate, based on the data points of a final part of the signal from 190 to 192 ps (Fig. 9b), that the principal frequency of compressive stress signal is approximately $11 \mathrm{THz}$, and our sampling frequency is approximately $100 \mathrm{THz}$ which corresponds to taking one sample every $\sim 9$

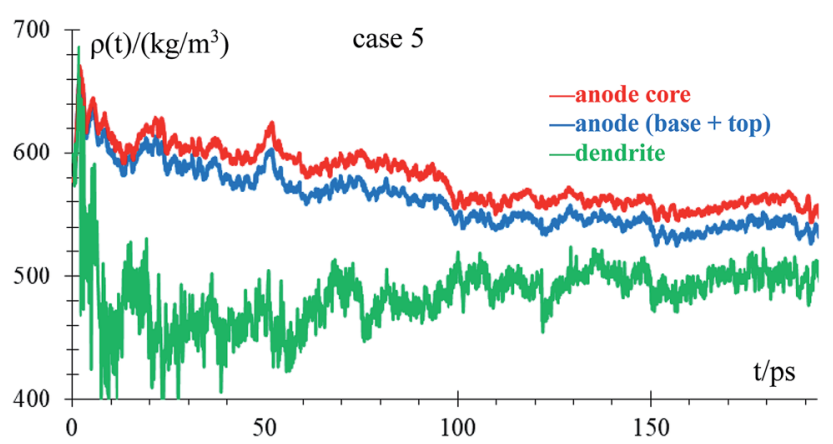

Fig. 8 Time evolution of the anode core density, dendrite, and whole anode. fs. Therefore, our sampling frequency follows quite well the shape of the signal. To confirm that our sample frequency is appropriate, we calculate the frequency spectrum of the compressive stress signal (Fig. 9c). The principal frequencies are below $15 \mathrm{THz}$ and after $40 \mathrm{THz}$ there are only noise as we are able to remake the original signal from the frequency spectrum up to $40 \mathrm{THz}$ yielding

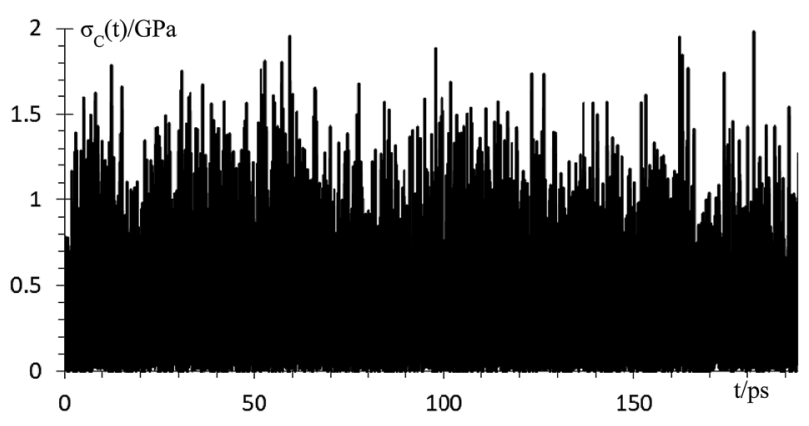

(a)

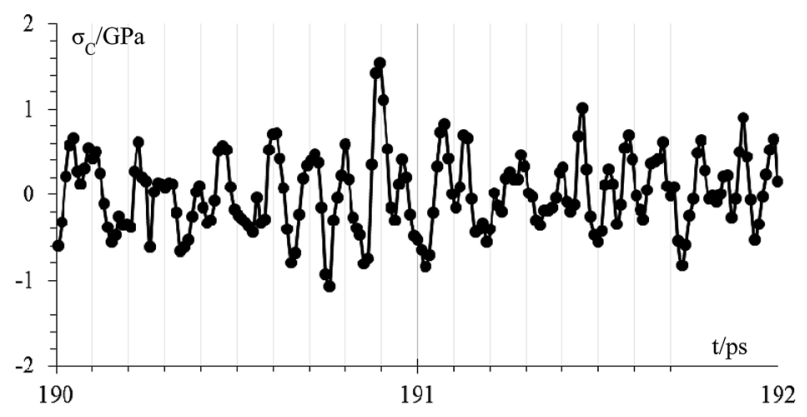

(b)

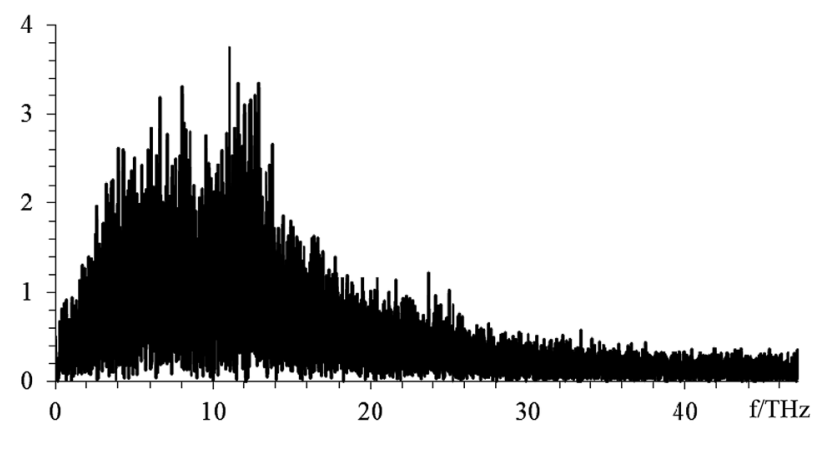

(c)

Fig. 9 (a) Compressive stress from Li-metal to electrolyte and SEI for case 5, (b) compressive stress from 190 ps to 192 ps, (c) frequency spectrum of the compressive stress signal. 

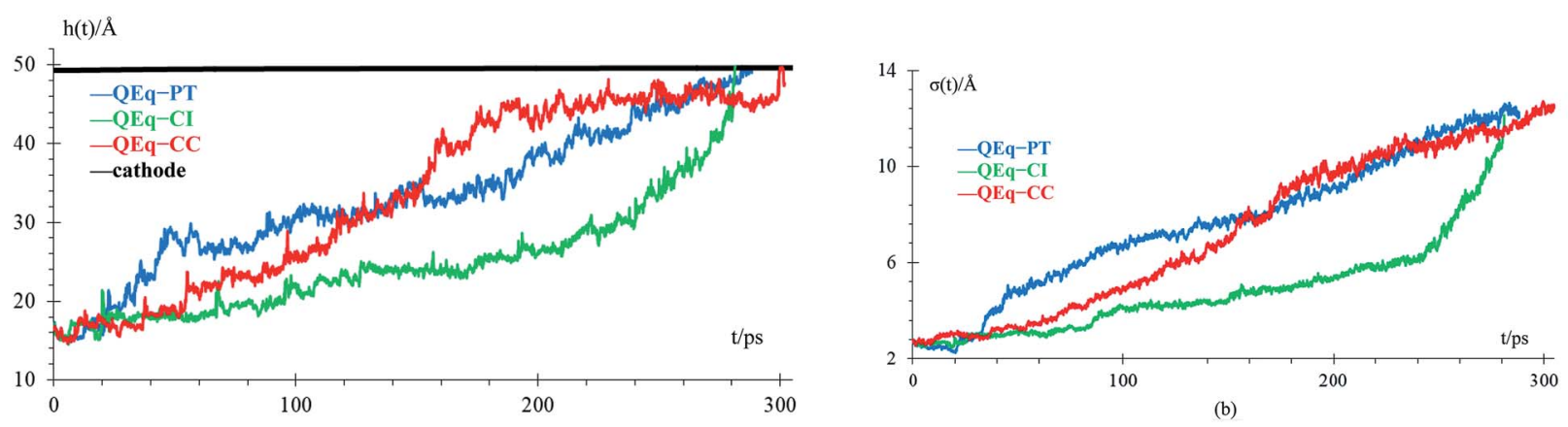

(a)
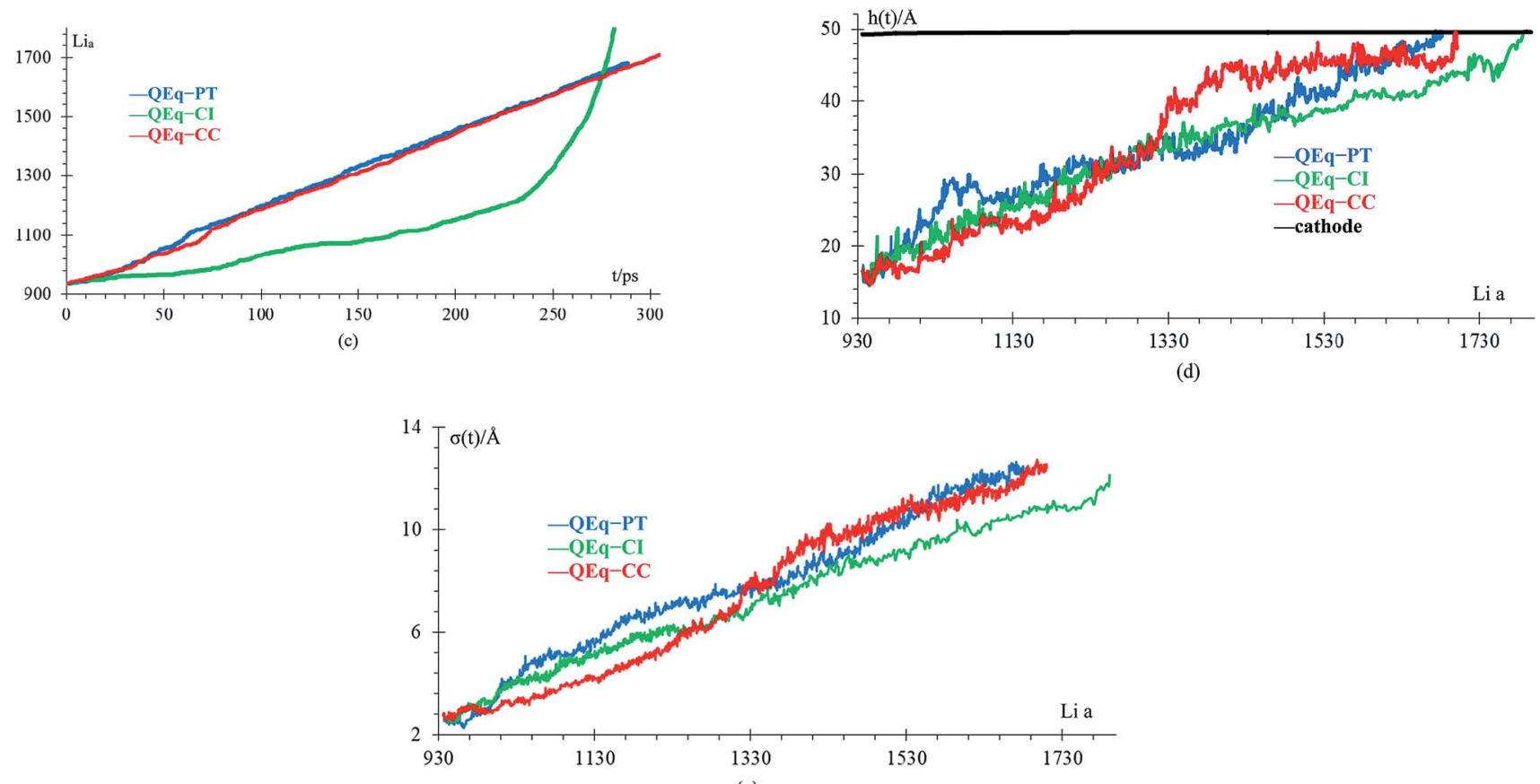

(e)

Fig. 10 (a) Height of the highest dendrite peak vs. time, (b) standard deviation of heights of lithium atoms in the anode surface vs. time, (c) number of lithium atoms in the anode $\left(\mathrm{Li}_{\mathrm{a}}\right) \mathrm{vs}$. time, (d) height of the highest dendrite peak vs. number of lithium atoms in the anode ( $\mathrm{Li}_{\mathrm{a}}$ ), (e) standard deviation of heights of lithium atoms in the anode surface vs. number of lithium atoms in the anode $\left(\mathrm{Li}_{\mathrm{a}}\right)$.

an error margin of less than $1 \%$; therefore, we can consider $40 \mathrm{THz}$ as the maximum frequency and as the sampling frequency of 100 $\mathrm{THz}$ is more than twice the maximum frequency of compressive stress, and according to Nyquist-Shannon sampling theorem, ${ }^{59}$ our sampling frequency is appropriate to represent in the frequency domain, the original time-domain signal.

We compare cases 6-8 using $Q_{\mathrm{Eq}}$ (Fig. 10), and we get that the amount of Li-metal before the short circuit at constant current is greater than at the pulse train, indicating that charging the battery at constant current is more effective than using a pulse train. In each case, a lithium dendrite grows. Li fills in the crack, even having a uniform lithiation, then forms a dendrite until the short-circuit takes place. The standard deviation of heights of lithium atoms in the anode surface also increases with time. All the behavior described before is the same as cases 1-5 (no $\left.Q_{\mathrm{Eq}}\right)$, which is not being changed by the electronic distribution. Consequentially, all results that we get for cases without $Q_{\mathrm{Eq}}$ would be similar if we made them with charge equilibration.
Finally, case 5 (std) and case $8\left(Q_{\mathrm{Eq}}-\mathrm{CC}\right)$ have the same lithiation rate of $1 \mathrm{Li}^{+}$every $0.4 \mathrm{ps}$, but the case $Q_{\mathrm{Eq}}-\mathrm{CC}$ can be charged more before the dendrite reaches the cathode. The main difference between these 2 cases is the use of charge equilibration, which facilitates the reaction in the electrolyte delaying the formation of lithium dendrites.

\section{Conclusions}

It is found that the electrolyte reactions delay the dendrite formation. Previous works showed that parts of the lithium dendrite break and remain in the electrolyte through charge/ discharge cycles resulting in the formation of dead $\mathrm{Li}^{60}$ In this work, we show that the dendrite is considerably more porous than the rest of the anode, allowing the formation of larger dendrites with low density and vulnerability to breaking, forming dead Li over multiple charge/discharge cycles. Also, the dendrite never grows spatially homogeneous even under uniform lithiation, but lithium always has a preference to 
deposit at specific parts of the anode, which causes the shape of the dendrite to change vertically with respect to the surface, increasing the distance between the anode peaks and anode valleys. We conclude that the mere presence of a cracked SEI greatly favors the lithium dendrite formation at the crack. Even if lithiation is uniform throughout the area, the lithium metal naturally will accumulate over the crack, forming a dendrite, and this takes place regardless of how the battery is charged. Certainly, LiF crack is the main driver for the growth of the dendrite. However, Li-ions also go through the uncracked areas of the $\mathrm{LiF}$ and get reduced and deposited on the metallic Lianode underneath, which is still covered by the LiF shell. These deposited $\mathrm{Li}$ increase the internal pressure toward further expansion and more cracking of the LiF shell and they actually contribute and help the growth of the dendrite from the bottom in addition to the growth from the top caused by those ions falling on top of the crack or of the growing dendrite tip. Finally, we estimated that the electrolyte must support a compressive stress of at least $2 \mathrm{GPa}$ to avoid the dendrite formation without suffering structural damage at $340 \mathrm{~K}$.

\section{Conflicts of interest}

There are no conflicts of interest to declare.

\section{Acknowledgements}

This material is based upon work supported by the U.S. Department of Energy's Office of Energy Efficiency and Renewable Energy (EERE), as part of the Battery 500 Consortium, Award Number DE-EE0008210. We also appreciate the support of computational resources from Texas A and M High Performance Research Computing (TAMU-HPRC) and the Texas Advanced Computing Center (TACC).

\section{References}

1 J. Lu, Z. Chen, Z. Ma, F. Pan, L. A. Curtiss and K. Amine, The role of nanotechnology in the development of battery materials for electric vehicles, Nat. Nanotechnol., 2016, 11(12), 1031.

2 W. Waag, C. Fleischer and D. U. Sauer, Critical review of the methods for monitoring of lithium-ion batteries in electric and hybrid vehicles, J. Power Sources, 2014, 258, 321-339.

3 S. Ziemann, D. B. Müller, L. Schebek and M. Weil, Modeling the potential impact of lithium recycling from EV batteries on lithium demand: a dynamic MFA approach, Resour., Conserv. Recycl., 2018, 133, 76-85.

4 C. Zhang, W. Lv, Y. Tao and Q.-H. Yang, Towards superior volumetric performance: design and preparation of novel carbon materials for energy storage, Energy Environ. Sci., 2015, 8(5), 1390-1403.

5 X. Zhang, X. Cheng and Q. Zhang, Nanostructured energy materials for electrochemical energy conversion and storage: a review, J. Energy Chem., 2016, 25(6), 967-984.
6 P. Bai, J. Li, F. R. Brushett and M. Z. Bazant, Transition of lithium growth mechanisms in liquid electrolytes, Energy Environ. Sci., 2016, 9(10), 3221-3229.

7 A. Zhamu, G. Chen, C. Liu, D. Neff, Q. Fang, Z. Yu, W. Xiong, Y. Wang, X. Wang and B. Z. Jang, Reviving rechargeable lithium metal batteries: enabling next-generation highenergy and high-power cells, Energy Environ. Sci., 2012, 5(2), 5701-5707.

8 C. Wang, Y. Yang, X. Liu, H. Zhong, H. Xu, Z. Xu, H. Shao and F. Ding, Suppression of Lithium Dendrite Formation by Using LAGP-PEO (LiTFSI) Composite Solid Electrolyte and Lithium Metal Anode Modified by PEO (LiTFSI) in AllSolid-State Lithium Batteries, ACS Appl. Mater. Interfaces, 2017, 9(15), 13694-13702.

9 W. Xu, J. Wang, F. Ding, X. Chen, E. Nasybulin, Y. Zhang and J.-G. Zhang, Lithium metal anodes for rechargeable batteries, Energy Environ. Sci., 2014, 7(2), 513-537.

10 G. Zheng, S. W. Lee, Z. Liang, H.-W. Lee, K. Yan, H. Yao, H. Wang, W. Li, S. Chu and Y. Cui, Interconnected hollow carbon nanospheres for stable lithium metal anodes, Nat. Nanotechnol., 2014, 9, 618.

11 D. E. Galvez-Aranda, V. Ponce and J. M. Seminario, Molecular dynamics simulations of the first charge of a Liion-Si-anode nanobattery, J. Mol. Model., 2017, 23(4), 120.

12 A. J. Bard, R. Parsons and J. Jordan, Standard Potentials in Aqueous Solution, CRC Press, 1985, vol. 6.

13 E. Peled, C. Menachem, D. Bar-Tow and A. Melman, Improved Graphite Anode for Lithium-Ion Batteries Chemically Bonded Solid Electrolyte Interface and Nanochannel Formation, J. Electrochem. Soc., 1996, 143(1), L4-L7.

14 H. Zheng, K. Jiang, T. Abe and Z. Ogumi, Electrochemical intercalation of lithium into a natural graphite anode in quaternary ammonium-based ionic liquid electrolytes, Carbon, 2006, 44(2), 203-210.

15 J. Cui, T.-G. Zhan, K.-D. Zhang and D. Chen, The recent advances in constructing designed electrode in lithium metal batteries, Chin. Chem. Lett., 2017, 28(12), 2171-2179.

16 C. Yang, K. Fu, Y. Zhang, E. Hitz and L. Hu, Protected Lithium-Metal Anodes in Batteries: From Liquid to Solid, Adv. Mater., 2017, 29(36), 1701169.

17 A. Jana and R. E. García, Lithium dendrite growth mechanisms in liquid electrolytes, Nano Energy, 2017, 41, 552-565.

18 P. Barai, K. Higa and V. Srinivasan, Lithium dendrite growth mechanisms in polymer electrolytes and prevention strategies, Phys. Chem. Chem. Phys., 2017, 19(31), 2049320505.

19 G. Gachot, S. Grugeon, M. Armand, S. Pilard, P. Guenot, J.-M. Tarascon and S. Laruelle, Deciphering the multi-step degradation mechanisms of carbonate-based electrolyte in Li batteries, J. Power Sources, 2008, 178(1), 409-421.

20 C. Zhang, Z. Huang, W. Lv, Q. Yun, F. Kang and Q.-H. Yang, Carbon enables the practical use of lithium metal in a battery, Carbon, 2017, 123, 744-755. 
21 Y. Guo, H. Li and T. Zhai, Reviving Lithium-Metal Anodes for Next-Generation High-Energy Batteries, Adv. Mater., 2017, 29(29), 1700007.

22 F. Wu, Y.-X. Yuan, X.-B. Cheng, Y. Bai, Y. Li, C. Wu and Q. Zhang, Perspectives for restraining harsh lithium dendrite growth: towards robust lithium metal anodes, Energy Storage Materials, 2018, 15, 148-170.

23 F. A. Soto, J. M. Martinez de la Hoz, J. M. Seminario and P. B. Balbuena, Modeling solid-electrolyte interfacial phenomena in silicon anodes, Curr. Opin. Chem. Eng., 2016, 13, 179-185.

24 X.-Q. Zhang, X. Chen, X.-B. Cheng, B.-Q. Li, X. Shen, C. Yan, J.-Q. Huang and Q. Zhang, Highly Stable Lithium Metal Batteries Enabled by Regulating the Solvation of Lithium Ions in Nonaqueous Electrolytes, Angew. Chem., Int. Ed., 2018, 57, 5301-5305.

25 A. Ramasubramanian, V. Yurkiv, T. Foroozan, M. Ragone, R. Shahbazian-Yassar and F. Mashayek, Lithium Diffusion Mechanism through Solid-Electrolyte Interphase in Rechargeable Lithium Batteries, J. Phys. Chem. C, 2019, 123(16), 10237-10245.

26 S. Plimpton, Fast parallel algorithms for short-range molecular dynamics, J. Comput. Phys., 1995, 117(1), 1-19.

27 W. M. Brown, P. Wang, S. J. Plimpton and A. N. Tharrington, Implementing molecular dynamics on hybrid high performance computers - short range forces, Comput. Phys. Commun., 2011, 182(4), 898-911.

$28 \mathrm{~W}$. M. Brown, A. Kohlmeyer, S. J. Plimpton and A. N. Tharrington, Implementing molecular dynamics on hybrid high performance computers - particle-particle particle-mesh, Comput. Phys. Commun., 2012, 183(3), 449459.

29 V. Ponce, D. E. Galvez-Aranda and J. M. Seminario, Analysis of a Li-Ion Nanobattery with Graphite Anode Using Molecular Dynamics Simulations, J. Phys. Chem. C, 2017, 121(23), 12959-12971.

30 J. M. Martinez de la Hoz, F. A. Soto and P. B. Balbuena, Effect of the Electrolyte Composition on SEI Reactions at Si Anodes of Li-Ion Batteries, J. Phys. Chem. C, 2015, 119(13), 70607068.

31 R. Payne and I. E. Theodorou, Dielectric properties and relaxation in ethylene carbonate and propylene carbonate, J. Phys. Chem., 1972, 76(20), 2892-2900.

32 Y. Chernyak, Dielectric Constant, Dipole Moment, and Solubility Parameters of Some Cyclic Acid Esters, J. Chem. Eng. Data, 2006, 51(2), 416-418.

33 D. E. Galvez-Aranda and J. M. Seminario, Simulations of a LiF Solid Electrolyte Interphase Cracking on Silicon Anodes Using Molecular Dynamics, J. Electrochem. Soc., 2018, 165(3), A717-A730.

34 M. J. Frisch, G. W. Trucks, H. B. Schlegel, G. E. Scuseria, M. A. Robb, J. R. Cheeseman, G. Scalmani, V. Barone, G. A. Petersson, H. Nakatsuji, X. Li, M. Caricato, A. Marenich, J. Bloino, B. G. Janesko, R. Gomperts, B. Mennucci, H. P. Hratchian, J. V. Ortiz, A. F. Izmaylov, J. L. Sonnenberg, D. Williams-Young, F. Ding, F. Lipparini, F. Egidi, J. Goings, B. Peng, A. Petrone, T. Henderson,
D. Ranasinghe, V. G. Zakrzewski, J. Gao, N. Rega, G. Zheng, W. Liang, M. Hada, M. Ehara, K. Toyota, R. Fukuda, J. Hasegawa, M. Ishida, T. Nakajima, Y. Honda, O. Kitao, H. Nakai, T. Vreven, K. Throssell, J. A. Montgomery Jr, J. E. Peralta, F. Ogliaro, M. Bearpark, J. J. Heyd, E. Brothers, K. N. Kudin, V. N. Staroverov, T. KeithR. Kobayashi, J. Normand, K. Raghavachari, A. Rendell, J. C. Burant, S. S. Iyengar, J. Tomasi, M. Cossi, J. M. Millam, M. KleneC. Adamo, R. Cammi, J. Ochterski, R. Martin, K. Morokuma, O. Farkas, J. Foresman and D. Fox, Gaussian 09; Revision A. 02, Gaussian. Inc., Wallingford, CT, USA, 2016.

35 L. Martínez, R. Andrade, E. G. Birgin and J. M. Martínez, PACKMOL: a package for building initial configurations for molecular dynamics simulations, J. Comput. Chem., 2009, 30(13), 2157-2164.

36 W. Humphrey, A. Dalke and K. Schulten, VMD: visual molecular dynamics, J. Mol. Graphics, 1996, 14(1), 33-38.

37 S. Alexander, Visualization and analysis of atomistic simulation data with OVITO-the Open Visualization Tool, Modell. Simul. Mater. Sci. Eng., 2010, 18(1), 015012.

38 Z. Cui, F. Gao, Z. Cui and J. Qu, A second nearest-neighbor embedded atom method interatomic potential for $\mathrm{Li}-\mathrm{Si}$ alloys, J. Power Sources, 2012, 207, 150-159.

39 H. Wang, X. Ji, C. Chen, K. Xu and L. Miao, Lithium diffusion in silicon and induced structure disorder: a molecular dynamics study, AIP Adv., 2013, 3(11), 112102.

40 L. A. Selis and J. Seminario, Dendrite formation in silicon anodes of lithium-ion batteries, $R S C A d v$., 2018, 8(10), 5255-5267.

41 K.-P. Huber, Molecular spectra and molecular structure: IV. Constants of diatomic molecules, Springer Science \& Business Media, 2013.

42 M. J. L. Sangster and M. Dixon, Interionic potentials in alkali halides and their use in simulations of the molten salts, $A d v$. Phys., 1976, 25(3), 247-342.

43 H. Luo, S. Xiao, S. Wang, P. Huai, H. Deng and W. Hu, Molecular dynamics simulation of diffusion and viscosity of liquid lithium fluoride, Comput. Mater. Sci., 2016, 111, 203-208.

44 M. M. Islam and A. C. T. van Duin, Reductive Decomposition Reactions of Ethylene Carbonate by Explicit Electron Transfer from Lithium: An eReaxFF Molecular Dynamics Study, J. Phys. Chem. C, 2016, 120(48), 27128-27134.

45 W. J. Mortier, S. K. Ghosh and S. Shankar, Electronegativityequalization method for the calculation of atomic charges in molecules, J. Am. Chem. Soc., 1986, 108(15), 4315-4320.

46 D. Bedrov, G. D. Smith and A. C. T. van Duin, Reactions of Singly-Reduced Ethylene Carbonate in Lithium Battery Electrolytes: A Molecular Dynamics Simulation Study Using the ReaxFF, J. Phys. Chem. A, 2012, 116(11), 2978-2985.

47 S.-P. Kim, A. C. T. v. Duin and V. B. Shenoy, Effect of electrolytes on the structure and evolution of the solid electrolyte interphase (SEI) in Li-ion batteries: a molecular dynamics study, J. Power Sources, 2011, 196(20), 8590-8597.

48 A. K. Rappe, C. J. Casewit, K. S. Colwell, W. A. Goddard and W. M. Skiff, UFF, a full periodic table force field for 
molecular mechanics and molecular dynamics simulations, J. Am. Chem. Soc., 1992, 114(25), 10024-10035.

49 N. Kumar and J. M. Seminario, Lithium-Ion Model Behavior in an Ethylene Carbonate Electrolyte Using Molecular Dynamics, J. Phys. Chem. C, 2016, 120(30), 16322-16332.

$50 \mathrm{~J}$. Haile and S. Gupta, Extensions of the molecular dynamics simulation method. II. Isothermal systems, J. Chem. Phys., 1983, 79(6), 3067-3076.

51 S. Nosé, A unified formulation of the constant temperature molecular dynamics methods, J. Chem. Phys., 1984, 81(1), 511-519.

52 M. R. Nadler and C. P. Kempier, Crystallographic Data 186. Lithium, Anal. Chem., 1959, 31(12), 2109.

53 A. Aryanfar, A. J. Colussi and M. R. Hoffmann, Lithium dendrite growth control using local temperature variation, Mater. Res. Soc. Symp. Proc., 2014, 1680.

54 C. T. Love, O. A. Baturina and K. E. Swider-Lyons, Observation of lithium dendrites at ambient temperature and below, ECS Electrochem. Lett., 2015, 4(2), A24-A27.
55 H. Yan, Y. Bie, X. Cui, G. Xiong and L. Chen, A computational investigation of thermal effect on lithium dendrite growth, Energy Convers. Manage., 2018, 161, 193-204.

56 D. Aurbach, E. Zinigrad, Y. Cohen and H. Teller, A short review of failure mechanisms of lithium metal and lithiated graphite anodes in liquid electrolyte solutions, Solid State Ionics, 2002, 148(3), 405-416.

57 D. Aurbach, E. Zinigrad, H. Teller and P. Dan, Factors Which Limit the Cycle Life of Rechargeable Lithium (Metal) Batteries, J. Electrochem. Soc., 2000, 147(4), 1274-1279.

58 L. Kong, Y. Xing and M. G. Pecht, In Situ Observations of Lithium Dendrite Growth, IEEE Access, 2018, 6, 8387-8393.

59 H. Nyquist, Certain topics in telegraph transmission theory, Trans. Am. Inst. Electr. Eng., 1928, 47(2), 617-644.

60 K.-H. Chen, K. N. Wood, E. Kazyak, W. S. LePage, A. L. Davis, A. J. Sanchez and N. P. Dasgupta, Dead lithium: mass transport effects on voltage, capacity, and failure of lithium metal anodes, J. Mater. Chem. A, 2017, 5(23), 11671-11681. 